Introduction Many studies have shown a link between exposure to pesticides and a higher level of DNA damage. However, longitudinal studies are rare and little is known about long term DNA damage and cancer risk in exposed subjects.

Objectives i) Quantify DNA damage for the women included in a French agricultural cohort at two time points, ii) study the relationship between DNA damage and agricultural tasks, and iii) between DNA damage and cancer incidence.

Methods 320 female agricultural workers were enrolled from 1997 to 2000 and completed a face-to-face questionnaire. 245 gave a blood sample at enrolment (T0). Ten years later, 104 donated another sample (T10). Using the comet assay with an internal historical negative control, we quantified DNA damage in PBMC on 200 nuclei using a 4-category visual scoring system.

Results At enrolment, there were no differences in DNA damage for farm owners/co-owners $(n=135,55 \%, p=0.84)$, or participants in livestock tasks within our study population $(\mathrm{n}=159,65 \%, \mathrm{p}=0.23)$. Pesticide exposure through application of anti-parasites to livestock $(n=138,56 \%, p=0.25)$ and disinfection of milking equipment $(n=117,48 \%, p=0.78)$ did not influence DNA damage. However, damage tended to be higher for those implicated in milking $(n=109,44 \%$, $\mathrm{p}=0.18)$. Women who carried out administrative $(\mathrm{n}=180$, $73 \%)$ and domestic tasks $(n=213,87 \%)$ tended to have lower damage scores $(\mathrm{p}=0.08$ and $\mathrm{p}=0.07$, respectively), as well as women who undertook other tasks had significantly lower damage $(\mathrm{p}=0.04)$ than those not involved. From enrolment to end of 2017, 37 incident cancer cases were diagnosed, of which 21 were breast cancer cases. No association was found between DNA damage and cancer incidence $(\mathrm{p}=0.45)$.

Conclusion Occupational exposure in the agricultural field could be evaluated using the comet assay. Women's involvement to tasks not related to farm upkeep show a lower level of DNA damage than those not undertaking these tasks.

\section{0-198 ALTERED LEVELS OF IMMUNE MARKERS AMONG MALE ROTATING NIGHT SHIFT WORKERS IN SPAIN - THE HORMONIT STUDY}

'Barbara Harding, Ruth Aguilar, Gemma Moncunill, Carlota Dobaño, Gemma Castaño Vinyals, Ana Espinosa, Kyriaki Papantoniou, Manolis Kogevinas. 'Barcelona Institute for Global Health, Spain

\subsection{6/OEM-2021-EPI.7}

Introduction Few preliminary studies suggest that night shift work is associated with a desynchronization of rhythmic immune parameters, which may explain in part the increased risk for infection, cardiometabolic disorders, and cancer in shift workers.

Objectives To examine how night shift work alters cellular immune markers.

Methods This study included 46 male rotating shift workers from a car industry in Barcelona, Spain, sampled twice toward the end of a 3-week night shift (22:00-06:00 hrs) and a 3week day shift (06:00-14:00 hrs) rotation. We collected four blood samples per worker; before and after during each shift (night shift at 06:00 and 14:00, day shift at 22:00 and 6:00). We measured 30 cytokines, chemokines and growth factors using Luminex technology and examined within-person variations in analytes between sampling time-points and shifts. We applied linear mixed models to examine within-person associations between shift and analytes, comparing samples taken at 6:00am during each shift. We also conducted a factor analysis using analyte levels from all 4 time points for each individual to identify common factors and determine if these factors were altered by shift work.

Results We observed lower levels of several analytes during the night shift (cytokines IL17, IL12, IL4, TNF $\alpha$, and cytokine receptors IL1RA, IL2R; chemokines IP10, MIP1 $\alpha$, MIP1 $\beta$, and RANTES; growth factors GCSF, FGF, EGF, HGF, and VEGF) compared to the day shift. In the factor analysis, the main factor (explaining 57\% of the variance) was negatively associated with night shift (beta: $-0.14,95 \% \mathrm{CI}-0.25$ to -0.03 , pvalue less than 0.01), indicating that night shift affects immune marker levels in this pathway. This factor included growth factors (FGF, EGF, HGF), Th1 type cytokines (IL15, IL2) and pro-inflammatory cytokines and chemokines (IL1 $\beta$, MIP1 $\alpha$, MIP1 $\beta)$.

Conclusion Our results show that night shift is associated with disruption in multiple immune response pathways.

\section{0-242 LITERATURE REVIEW OF BIOLOGICAL MONITORING STUDIES TO ASSESS HEALTHCARE WORKER'S EXPOSURE TO ANTINEOPLASTIC DRUGS}

${ }^{1}$ Naqiyah Motiwala, Chun-Yip Hon. ${ }^{1}$ Ryerson University, Canada

\subsection{6/OEM-2021-EPI.8}

Introduction Antineoplastic drugs, also known as hazardous drugs, are used to treat cancer. Healthcare workers' exposure to these agents may lead to DNA damage and adverse reproductive effects. Exposure to antineoplastic drugs can be assessed via biological monitoring.

Objectives To conduct a literature review of studies examining biological monitoring of healthcare workers exposed to antineoplastic drugs to identify trends and knowledge gaps.

Methods The literature search was performed on three databases: Proquest, Web of Science and PubMed. For inclusion, an article had to be in English and published between 2005 and 2020. Search terms included 'antineoplastic drugs' or 'hazardous drugs' AND 'occupational' or 'healthcare workers' or 'pharmacist' or 'nurse' AND 'blood' or 'urine' or 'biomarkers'.

Results Overall, the literature review resulted in 70 unique articles - 30 examined urine as the matrix, 31 had non-urine samples and nine papers collected both urine and non-urine samples. Regarding the urine sampling studies, researchers collected either spot samples or 24-hr voids. Cyclophosphamide was the most common analyte (69\%) with a detection limit ranging from 0.09 to $0.20 \mathrm{ng} / \mathrm{ml}$. Approximately $75 \%$ of the articles reported at least one urine sample had detectable levels of drug. In some instances, urinary drug contamination was found even though workers wore personal protective equipment. For those studies that collected other matrices, blood and exfoliated buccal cells were the most common specimens. A majority (81\%) reported that there was a statistically significant difference in results between exposed and non-exposed populations. Many studies reported that safe work practices, which includes the use of personal protective equipment, may not be adequate to protect healthcare workers. 
Conclusion There is value in conducting biological monitoring to assess healthcare workers' exposure to antineoplastic drugs. However, because of the variability in sampling and analytical methods, standardization is recommended to ensure biological monitoring results are comparable.

\section{0-355 THE BIOCHEMICAL EFFECTS OF LEAD CONCENTRATION ON OXIDATIVE STRESS PARAMETERS IN WORKERS}

'Mehrzad Ebrahemzadih, Hamzeh Salehzadeh. 'Neyshabur University of Medical Sciences, Islamic Republic of Iran

\subsection{6/OEM-2021-EPI.9}

Objective The goal of this study was to determine the blood lead concentration in various occupational workers in Sanandaj and its effects on oxidative stress parameters and other blood parameters.

Methods This descriptive-analytical study was carried out in Sanandaj, Iran. A total of 278 subjects were selected as the sample size for this study, of which 64 did not have exposure to occupational lead, and 214 had occupational exposure to lead. The blood lead levels were measured by the atomic absorption spectrophotometer apparatus AA6800. Oxidative stress parameters and other blood parameters were measured by special devices.

Results and Conclusion There was a significant positive relationship between the blood lead concentration and the oxidative stress parameters including lipid peroxidation, malondialdehyde, glutathione, antioxidant serum, and catalase. There was a significant negative relationship between the blood lead concentration and hematological parameters. Also, there was a significant positive relationship between the blood lead level and the lead concentration in the air.

\section{0-399 URINARY PESTICIDE METABOLITE LEVELS AMONG FARM WORKERS IN MALAYSIA: PILOT RESULTS FROM THE IMPRESS STUDY}

'Zulkhairul Naim Bin Sidek Ahmad, Anne-Helen Harding, Hans Kromhout, loannis Basinas, John Cherrie, Karen Galea, Kate Jones, Martie van Tongeren, Roel Vermeulen, Samuel Fuhrimann, Will Mueller, Andrew Povey. ${ }^{1}$ China Medical University, Taiwan

\subsection{6/OEM-2021-EPI.10}

Introduction Pesticides are widely used by farmers in Malaysia but there is limited information on exposures experienced.

Objective To determine Malaysian farmer's exposure from pesticide spray events through the collection and analysis of urine samples.

Method 25 farmers growing either rice $(n=16)$ or vegetables/ fruits $(n=7)$ from the east coast of Malaysia provided two urine samples, one in the morning before they started spraying and the other in the evening of the first day of spraying either with chlorpyrifos $(n=16)$, cypermethrin $(n=18)$ or pyrethroids $(n=21)$. Urinary levels of 3,5,6-Trichloro-2-pyridinol (TCPy), 3-phenoxybenzoic acid (3PBA), and total 2,2, dimethylcyclopropane carboxylic acid (DCVA) were determined by liquid chromatography-mass spectrometry and corrected for urinary creatinine levels.
Results Farmers who used the precursor pesticide had higher levels of urinary metabolites after spraying than those who did not. Urinary TCPy levels of chlorpyrifos applicators were significantly higher post-spraying compared to pre-spraying: median levels and interquartile range (IQR) were 94 (18.6$228.4)$ versus $43(18.8-121.5) \mu \mathrm{mol} / \mathrm{mole}$ creatinine respectively ( $p$ urinary 3PBA levels of pyrethroid users $(n=21)$ were also significantly higher post-spraying than pre-spraying: median levels (IQR) were $1.8(0.6-3.7)$ versus 0.9 (0.5-1.7) $\mu \mathrm{mol} / \mathrm{mole}$ creatinine ( $\mathrm{p}$ Urinary DCVA levels of cypermethrin users $(n=18)$ were significantly higher post-spraying than prespraying : median levels (IQR) were 5.6 (2.3-10.3) versus 1.9 (1.4-4.2) $\mu \mathrm{mol} / \mathrm{mole}$ creatinine (p Pre-spraying urinary TCPy (but not 3PBA or DCVA) levels in farmers who had applied the precursor pesticide were higher than pre-spray levels in farmers who had not $(\mathrm{p}<0.01)$.

Conclusion Current use of pesticides by Malay farmers results in increased exposure that can be detected by urinary metabolite measurement. This suggests further training is needed to reduce exposure in this population.

\section{Breast Cancer}

\section{0-10 BREAST CANCER IN DANISH WOMEN OCCUPATIONALLY EXPOSED TO DIESEL EXHAUST AND POLYCYCLIC AROMATIC HYDROCARBONS}

'Julie Pedersen, Katrine Strandberg-Larsen, Michael Andersson, Johnni Hansen. 'Danish Cancer Society, Denmark

\subsection{6/OEM-2021-EPI.11}

Introduction Both diesel exhaust and specific polycyclic aromatic hydrocarbons (PAHs) have been classified as carcinogenic to humans. However, there is overall limited evidence for an association between these exposures and breast cancer. Objectives To explore the association between occupational exposure to diesel exhaust and PAHs, respectively, and breast cancer, including subtypes.

Methods The study included 38,375 women with incident breast cancer identified in the Danish Cancer Registry, and five breast cancer-free controls per case matched on year of birth who were randomly selected from the Danish Civil Registration System. Full employment history was obtained for all women from a nationwide pension fund, and exposure to diesel exhaust and PAHs was assessed using a job exposure matrix. Conditional logistic regression was used to estimate of odds ratios (ORs) with adjustment for reproductive factors and socioeconomic status.

Results No noteworthy associations were observed for overall breast cancer in women exposed to diesel exhaust. However, diesel exhaust modestly elevated the risk of estrogen receptor negative breast tumours before age 50 years $(\mathrm{OR}=1.26,95 \%$ CI: 1.09-1.46). Duration- and dose-response relationships were also observed for this subtype in this age group. No notable risk patterns were generally observed for $\mathrm{PAH}$ exposure.

Conclusion Occupational exposure to diesel exhaust may elevate the risk of early-onset estrogen receptor negative breast tumours in women. Future studies exploring this association are warranted. 